\title{
DESIGN OF SIMULATION STRATEGY FOR CORN CLEANING ON DISCRETE ELEMENT METHOD
}

\author{
Hua $\mathrm{Li}^{1}$, Hongju Gong ${ }^{1}$, Wenqing Yin ${ }^{1, *}$ \\ 1 College of Engineering Nanjing Agricultural University, Nanjing Jiangsu province, \\ P.R.china 210031 \\ * Corresponding author,Address:College of Engineering Nanjing Agricultural University, \\ Nanjing 210031 Jiangsu province,P.R.china.Tel:+86-25-58606607,Fax:+86-25-58606585, \\ E-mail:lihua@njau.edu.cn
}

\begin{abstract}
The past efforts made on the corn cleaning dynamics were reviewed. Based on Discrete Element Method model, a simulation method for corn cleaning dynamics was then proposed, composing the following design factors: 1. 2-D discrete model and its related factors; 2. parities and magnitude of computation, consideration of the two factors upon application; 3 . test platform design. The implementation of this plan will illustrate the dynamics of corn cleaning, benefiting the efficiency of cleaning machine design and the improvement of production economies.
\end{abstract}

Keywords: Discrete element method (DEM), corn cleaning, strategy, simulation

\section{INTRODUCTION}

Cleaning is one important step of corn harvesting. The earliest record on cleaning dated back to the Spring and Autumn period in China, in which manual sieve and windmill were used. The modern combine harvesters applied both wind cleaning and sieve cleaning. And the repeated revision on the design work for the configuration and key parts have been made by agricultural engineers, with an aim of reducing energy demand.

Please use the following format when citing this chapter:

Li, H., Gong, H. and Yin, W., 2009, in IFIP International Federation for Information Processing, Volume 293, Computer and Computing Technologies in Agriculture II, Volume 1, eds. D. Li, Z. Chunjiang, (Boston: Springer), pp. 603-611. 
Dynamics of corn cleaning still remains as a complex problem. The mainstream efforts of research are still concerned with pneumatic reciprocal sieve mechanism, and mostly are of parameterized characterizes, e.g., adjusting physical parameters of processed materials and changing construction and motion to illustrate its cleaning performances. The commonly used theory is particle dynamics, in which the whole grain collection is taken as a single particle. And trajectory tracing and high-speed photography have also been adopted to quantify the cleaned grain (Fang et al., 1999; Ge et al., 2001; Jianping et al., 1997; Wenqing et al., 2002; Wenqing et al., 2001). In the cleaning procedure, grain behaves much like that of a fluid, and thus a precise description of its state is a primary problem.

DEM was proposed by Cundal in 1970's (PA et al., 1979), which fundamental concept was to take the discrete grains as a collection of particles with particular shape and mass. And contacting mechanics model was assigned to the neighboring surfaces between particles and boundaries or between particle and particles. Wang introduced DEM into China and quickly used this theory in soil mechanics (Yongjia et al., 1991; Jibo et al., 1990). And Thornton DEM was applied by China agricultural university in 1998 for simulation (Yong et al., 1999).

Flowing is a typical property of grain as it is transferred to platy shaking sieve in cleaning. The grain collection is not a continuum substance, and force interaction exists among grain particles. Thus taking grain collection as a whole will introduce error for simulation, making it impossible for a correct interpretation of interactions among particles. Compared with this, DEM is much better. Sakaguchi and Suzuki et al. investigated the vibration cleaning process of paddy and rice with DEM (E et al., 2001). Introduced a 2-D plate model with relative parameters, A DEM simulation strategy was supplied and then implemented.

\section{THE BASIC IDEAS OF SIMULATION ON DEM}

The basic idea of DEM simulation on grain cleaning provided in this paper is to apply a micro-mechanics method, i.e. study on particle-particle and particle-boundary interactions. By providing correct contacting mechanics model and supplying governing equations, calculation with rational algorithms is performed on work station to provide dynamic parameters such as force, displacement, deformation and velocity. The ontime simulation serves as a complementary method for scientific design on cleaning mechanisms, for innovative theory, calculation and assessment, which main principles include (Yong et al., 2003):

1) Grain particle was taken as a 2-D platy element. To achieve a precise simulation, soybean was used in experiment to illustrate layering and sieving 
performances of grain on platy sieve. Sieve surface was simplified as a plane, and sieve openings were simplified as virtual boundary. When the contacting width between grain particle and virtual boundary reaches $2 \mathrm{R}$, the grain will pass the sieve.

2) Visco-elastic model was used for grain interaction modeling to analyze normal and tangential contacting force. The amount of normal overlaying is the difference between radius and center-to-center distance, otherwise it is regarded non-contacting.

3) Central differential method was applied with small time elapse to calculate kinetic differential equations, giving the displacement and velocity of each particle in each instant.

4) DEM simulation was then conducted with reference to displacement and velocity table of particle collection.

5) Result was laboratory tested with high-speed camera recording and compared with simulation. By adjusting experimental parameters a satisfactory result was achieved.

\section{DESIGN OF SIMULATION PROGRAM FOR GRAIN CLEANING}

\subsection{Discrete element model of grain in cleaning}

Analytical model should be constructed before DEM simulation. In addition to the requirement of the geometrical approximation of the model grain to that of the real one, factors must be considered for the judgment of the contacting condition between neighboring particles or particle and boundaries, and for the simplicities of the calculation of the overlaying magnitude, so that the computing capacity of the computer is satisfied and certain precision of calculation is balanced.

Based on the mechanics and contacting mode of discrete materials, the underlining models are listed in table 1

Table 1. Analysis of the various particles of the main features of model

\begin{tabular}{lcccl}
\hline \multicolumn{1}{c}{ Types of particles } & Physical characteristic & Contact judgment & Computation & Applicability \\
\hline ball-particle & better & easy & relatively less & sand, soybean etc. \\
ellipsoid -particle & excellent & complex & heavy & rice etc. \\
ball-column & excellent & difficult & heavy & some soil particles etc. \\
hypersphere model & excellent & difficult & heavy & complicated grains etc. \\
combination ball model & good & difficult & heavy & nail grains etc. \\
\hline
\end{tabular}


Considering laboratory test and theoretical research, 2-D platy model of particle were applied, which is illustrated in fig. 1.

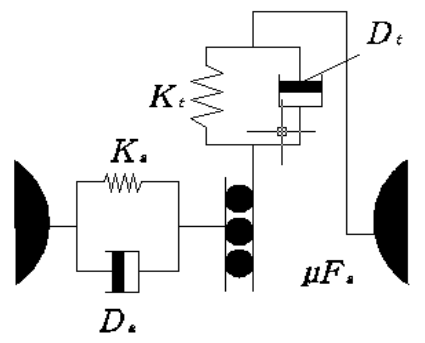

Fig.1: Contact model between particles

Fundamental parameters in the applying model were measured and provided in table 2 .

Table.2 The main physical parameters

\begin{tabular}{cccccc}
\hline 1000-grain weight & $\begin{array}{c}\text { Friction } \\
\text { coefficient }\end{array}$ & $\begin{array}{c}\text { Contact stiffness } \\
\text { (particle-particle })\end{array}$ & $\begin{array}{c}\text { Contact stiffness } \\
\text { (particle-border) }\end{array}$ & $\begin{array}{c}\text { Damping } \\
\text { coefficient }\end{array}$ & $\begin{array}{c}\text { Recovery } \\
\text { coefficient }\end{array}$ \\
\hline $49.5(\mathrm{~g})$ & $0.402(\mathrm{~N} / \mathrm{s})$ & $25000(\mathrm{~N} / \mathrm{s})$ & $45000(\mathrm{~N} / \mathrm{s})$ & $0.2(\mathrm{Ns} / \mathrm{m})$ & 0.7 \\
\hline
\end{tabular}

Elasto-damp-friction model was applied on the contacting between particle and sieve (Asmar, 2002; Anyuan et al., 2002), which is shown in fig.2.

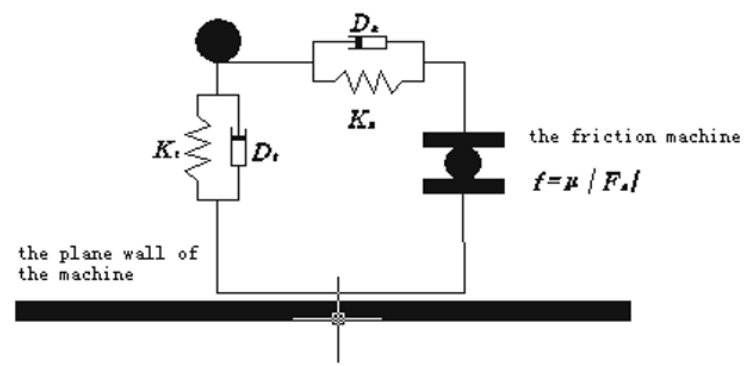

Fig. 2 :contact model between particle and sieve plate

One assumption of the model is the point contact between ball particle and sieve surface, allowing certain amount of overlaying in colliding. Contacting force is determined by both the magnitude of overlaying and the relative velocity of the two.

Contacting force is generally considered as the addition of elastic force and damping force, which are calculated in the following equations (Hongguan et al., 2006) :

$$
\mathrm{F}_{\mathrm{e}}=\mathrm{k} * \delta
$$




$$
\mathrm{F}_{\mathrm{d}}=\mathrm{D} * \mathrm{~V}
$$

Thereinto, $\mathrm{K}$ is the contact stiffness between particles and the wall of container, $\delta$ the composite quantity between them, $\mathrm{V}$ the relative velocity for the particles and the wall at the contact points, and D the damping coefficient between them, D is computed according the following formulas:

$$
\begin{aligned}
& D=2 \gamma \sqrt{m_{p s} * k} \\
& \gamma=-\frac{\ln (e)}{\sqrt{\pi^{2}+\ln ^{2}}(e)} \\
& m_{p s}=\frac{m_{p} \cdot m_{s}}{m_{p}+m_{s}}
\end{aligned}
$$

Thereinto, e is the recovery factor for the collision between particles and the plane, $m_{p}$ is the quality of the particle, and $m_{s}$ is the quality of sieve surface. Divide the contact force into normal and tangential contacting force in the actual calculation, and compute respectively in the form of incremental direction.

\subsection{Consideration of simulation accuracy}

It is not easy to simulate the grain cleaning with DEM. In order to reach a certain simulate accuracy, scientific and careful consideration is indispensable. The current level both at home and abroad only rest on the reasonable characterization and tending to be similar. When simulating with DEM, we seize the main factors so that simulation is right in the shape firstly and then explore in depth to make the simulation accurate in essence. The important questions we have to consider are as following:

1) The equivalent of particle (Yong et al., 2003)

The analysis and simulation of the grain cleaning need to study corresponding relationship between particles and the model. We can get the rational parameters of the model according to the physical properties of particles practically. The main considerations dealt with equivalent in this study are:

(1) The principle of equal quality

In the calculation area, the total quality of grain should be equal to the particle groups in the model, and at the same time, the total quality of grain should be equal to the particle groups when they are equal in the volume, that is they have the same density, so in this way the mechanical responding concerned to the gravity can be assured in the mass.

(2) The principle of equivalent fluidity for particles 
With the actual grain geometry model having a great gap with the ballmodel, the stream of grain in the sieve surface also is varies with the fluidity of the model, so it is need to modify the parameters of that model in order to make the model close to reality as far as possible. We should adjust the particle-friction coefficient and adhesion surface energy so that the ballmodel and the corresponding tests have the same rest angles, which is the typical index indicating the friction property and fluidity of discrete material.

2) Calculating scale

Because the computation load will increase in the way of geometric series when increasing the number of particles, so a very high capability for computer is demanded. At the same time, the effect of simulation will decrease sharply if the number of particles reduces. Therefore, we choose a relatively rapid one after studying much arithmetic and recur to the advanced computing platforms and graphical interface, so that a better simulation outcome can be gained.

\subsection{The simulation process with DEM}

With the DEM and programming tools, the analysis program of DEM can be written out. The flow chart of programme is as follows:

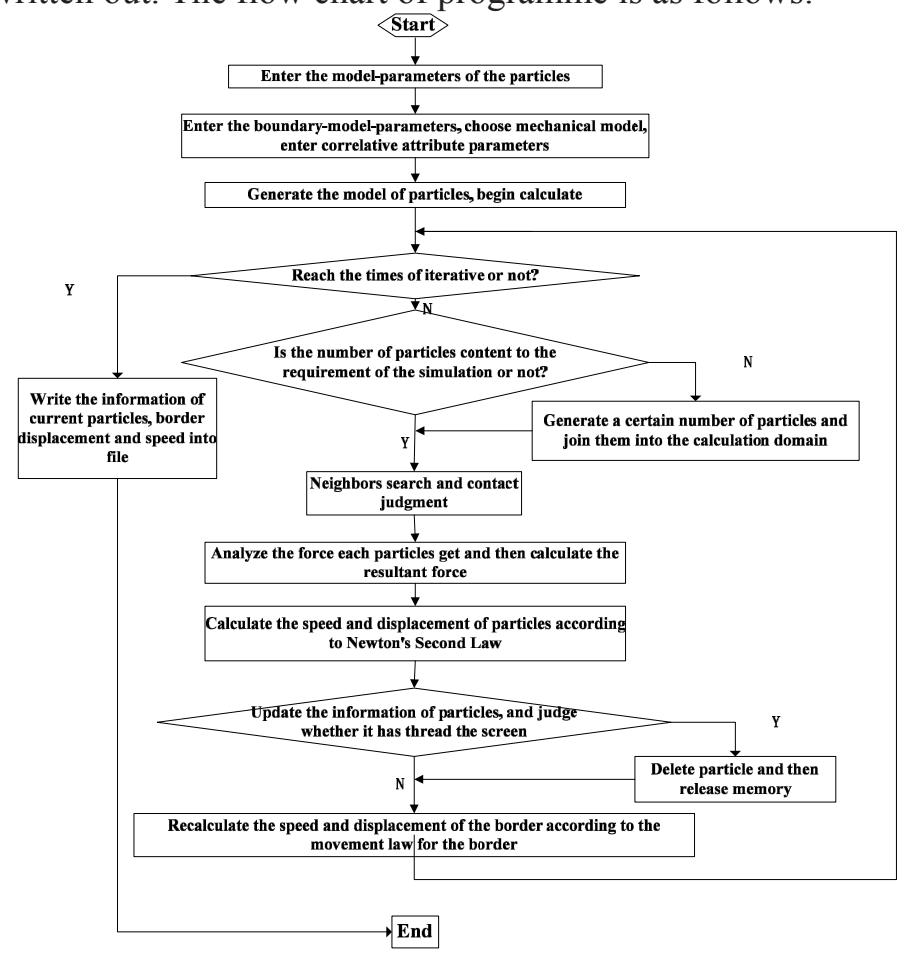

Fig.3: flow chart based on DEM 


\subsection{Test verification}

The simulation of the corn cleaning is needed to be verified .Test verification is mainly as follows:

1) Measure the physical characteristics of grain

In order to make the particle model and the actual equivalent similar as far as possible, the physical characteristics of grain muse be measured such as the 1000-grain weight, friction coefficient, contact stiffness (particle particle and particle-border), the recovery coefficient and the rest angles etc.

2) Verify the simulation results in the test platform

Whether the simulation graphics based on DEM is vivid of not, the results will be verified by the experiment in the test platform. According to the configuration of combine, we have designed the general experimental systems, which is composed of plane vibration system and high-speed motion analysis system(shown in Figure 4). In it,

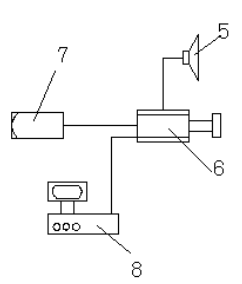

(a)High-speed motion analysis system

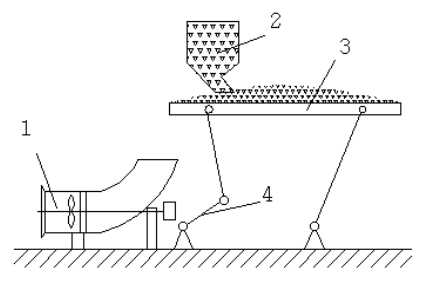

(b)Plane vibration system

Fig.4: The experimental systems

1-fan 2-feeding device 3-vibration sieve 4- motor 5- high-power light source 6- high-speed camera 7-mobile HD 8-computer

It is designed specially for studying the trajectory of materials. The device mainly includes gantry, wobbling boards, screen, fan, motor, inverter and sampling box.

In the device, the length of the cleaning sieve is $1000 \mathrm{~mm}$, width $300 \mathrm{~mm}$, and they can be changed when flat board or through sieve is needed. The sieve works in the form of linear reciprocating vibration driven by Crank Linkage.

The centrifugal fan is used. Type: YDF - 2.24B-6S; Standard rated speed: $960 \mathrm{r} / \mathrm{min}$; Flux: $1300 \mathrm{~m}^{3} / \mathrm{h}$. Adjust the speed of fan, the speed of air current can be changed.

Y-type alternating current stepper motor is selected. It's rated voltage is $220 \mathrm{~V}$, rated power $0.75 \mathrm{KW}$, and rated speed $1440 \mathrm{r} / \mathrm{min}$. The speed of driven motor should be adjusted according to the need of requirements. 
The inverter is called as BT40D digital transducer.

The two sides of the cleaning room are made respectively of organic glass and white cardboard. The adoption of organic glass is for the sake of visualization research. The vidicon can record the motive scene through the organic glass. Using the white cardboard is in favor of enhancing the contrast of backgrounds and objectives and is conducive to the following image processing.

The MVC1000SAGE30 high-speed camera produced by Microview Company is used in this test.

\section{CONCLUSIONS}

Because the grain groups own the characteristics of multiphase, dispersing, kinetic and randomicity and so on in the cleaning process, its complicated kinetic and mechanical specialties bring a great obstacle to the improvement and innovation of harvest machines. Based on the research outcomes of predecessors and combined the properties of corn sieve, the simulation strategy for corn cleaning on DEM is carried out, This project is implementing in our laboratory.

The simulation strategy is also applicable to other analysis of discrete element. Combining the DEM and the configuration of grain cleaning and then simulating in computer, the design efficiency for the grain cleaning machine and economy of the products can be improved greatly.

\section{REFERENCES}

Anyuan Li, Shi Liu,Zhonggang Pan. Numerical Simulation of Movement of Solid Particles in a Vibrating Feeder[J]. Journal of the Graduate School of the Chinese Academy of Sciences. 2002,19 (1): 35-42.

Asmar B. N. Valiadation tests on a distinct element model of vibrateing eohesive particle systems[J]. Computer and Chemical Engineering. 2002,26 : 785-802.

E Sakaguchi, M Suzuki,F Favier J. Numerical simulation of the shaking separation of paddy and brown rice using the discrete element method[J]. J Agric EngngRes. 2001,79 (3): 307315.

Fang Cheng,Jun Wang. Test Study on Main Parameters 0t Air-and-Screen Cleaning Mechanism[J]. Transactions of the CSAE. 1999,15 (1): 55-58.

Ge Li, Yun Zhao,Gaohong Yu. Dynamical Property, Loci and Separating of Materials in Inclined Airflow Device[J]. Transactions of the CSAE. 2001,17 (6): 22-25.

Hongguan Jiao,ZhaoYaoming. Computer emulation of particle motion on the screen plate and its validation by test[J]. Mining and metallurgy. 2006,15 (1): 63-67.

Jianping Li,Yun Zhao. Experiment and Computer Simulation of Tossing Motion of Agricultural Materials on Oscillating Sieve[J]. Transactions of the CSAE. 1997,13 (4): 4665 . 


\section{Method}

Jibo Xing,Yongiia Wang. Improvement of Discrete Element Method and its Applications in Granular Media[J]. Journal of Geotechnical Engineering. 1990,12 (5): 51-57.

PA Cundall,StrackOL. A discrete numerical model for granular assembles[J]. Geotechnique. 1979,29 (1): 47-65.

Wenqing Yin, Kutzbach H. D. Experimental Research on Cleaning Shoe with Circular Vibrating Sieve[J]. Transactions of the CSAE. 2002,18 (6): 81-83.

Wenqing Yin, Peter Wacker,Kutzbach Heinz Dieter. Maehdrescher-Reinigungsanlage[J]. Landtechnik. 2001,(4): 276-277.

Yong Xu, D Kafui K.,C Thornton. Silo Discharge Simulations With Different Particulate Properties Using the Distinct Element Method[J]. Transactions of the CSAE. 1999,15 (3): 65-69.

Yong Xu, Hongyan Li,Wenbin Huang. Modeling and methodological strategy of discrete element method simulation for tillage soil dynamics[J]. Transactions of the CSAE. 2003,19 (2): 34-38.

Yongjia Wang,Jibo Xin . Discrete element method and its application in rock mechanics[M]. Shengyang, Northeast engineering institute Press,1991: 